\title{
Genome-wide analysis of the PreA/PreB (QseB/QseC) regulon of Salmonella enterica serovar Typhimurium Massimo Merighi ${ }^{\dagger 1,5}$, Alecia N Septer ${ }^{\dagger 1,7}$, Amanda Carroll-Portillo ${ }^{2,6}$, Aditi Bhatiya1, Steffen Porwollik ${ }^{3}$, Michael McClelland ${ }^{3}$ and John S Gunn*1,4
}

\begin{abstract}
Address: ${ }^{1}$ Center for Microbial Interface Biology and Department of Molecular Virology, Immunology and Medical Genetics, The Ohio State University, 333 W. 10thAvenue, Columbus, OH 43210, USA, 2University of Texas Health Science Center at San Antonio, 7703 Floyd Curl Dr, San Antonio, TX 78229, USA, ${ }^{3}$ Sidney Kimmel Cancer Center, 10835 Road to the Cure, La Jolla, CA 92121, USA, ${ }^{4} 1006$ BRT, 460 W. 12 th Avenue, Columbus, OH 43210, USA, 5 Department of Microbiology and Molecular Genetics, Harvard Medical School, Boston, MA 02115, USA, ${ }^{6}$ Department of Biomolecular Materials and Interfaces, Sandia National Laboratories, Albuquerque, NM 87185, USA and ${ }^{7}$ Department of Microbiology, University of Georgia, Athens, GA 30602, USA

Email: Massimo Merighi - massimo_merighi@hms.harvard.edu; Alecia N Septer - ansepter@gmail.com; Amanda CarrollPortillo - acarrol@sandia.gov; Aditi Bhatiya - aditi.bhatiya@osumc.edu; Steffen Porwollik - sporwollik@skcc.org; Michael McClelland - mmcclelland@skcc.org; John S Gunn* - gunn.43@osu.edu

* Corresponding author †Equal contributors
\end{abstract}

Published: 23 February 2009

BMC Microbiology 2009, 9:42 doi:10.1186/147|-2180-9-42
Received: I October 2008

Accepted: 23 February 2009

This article is available from: http://www.biomedcentral.com/I47I-2/80/9/42

(C) 2009 Merighi et al; licensee BioMed Central Ltd.

This is an Open Access article distributed under the terms of the Creative Commons Attribution License (http://creativecommons.org/licenses/by/2.0), which permits unrestricted use, distribution, and reproduction in any medium, provided the original work is properly cited.

\begin{abstract}
Background: The Salmonella PreA/PreB two-component system (TCS) is an ortholog of the QseBC TCS of Escherichia coli. In both Salmonella and E. coli, this system has been shown to affect motility and virulence in response to quorum-sensing and hormonal signals, and to affect the transcription of the Salmonella enterica serovar Typhimurium (S. Typhimurium) pmrAB operon, which encodes an important virulence-associated TCS.
\end{abstract}

Results: To determine the PreA/PreB regulon in $S$. Typhimurium, we performed DNA microarrays comparing the wild type strain and various preA and/or preB mutants in the presence of ectopically expressed preA (qseB). These data confirmed our previous findings of the negative effect of PreB on PreA gene regulation and identified candidate PreA-regulated genes. A proportion of the activated loci were previously identified as PmrA-activated genes (yibD, pmrAB, cptA, etc.) or were genes located in the local region around preA, including the preAB operon. The transcriptional units were defined in this local region by RT-PCR, suggesting three PreA activated operons composed of preA-preB, mdaB-ygiN, and ygiW-STM3175. Several putative virulence-related phenotypes were examined for preAB mutants, resulting in the observation of a host cell invasion and slight virulence defect of a preAB mutant. Contrary to previous reports on this TCS, we were unable to show a PreA/PreB-dependent effect of the quorum-sensing signal Al-2 or of epinephrine on $S$. Typhimurium with regard to bacterial motility.

Conclusion: This work further characterizes this unorthadox OmpR/EnvZ class TCS and provides novel candidate regulated genes for further study. This first in-depth study of the PreA/PreB regulatory system phenotypes and regulation suggests significant comparative differences to the reported function of the orthologous QseB/QseC in E. coli. 


\section{Background}

Salmonella spp. have a broad host range and antibiotic resistant isolates are on the rise [1]. Salmonellae infections of humans result in two primary clinical manifestations: enteric (typhoid) fever and gastroenteritis. The latter is characterized by a local infection primarily of the small intestine and involves massive neutrophil transmigration into the intestinal lumen. Typhoid fever is a systemic infection in which the bacterium is carried from the intestinal submucosa to distal organs primarily within host cells such as macrophages.

Two-component signal transduction is critical for the adaptation of Salmonella enterica serovar Typhimurium ( $S$. Typhimurium) to the diverse array of environments encountered outside and inside its hosts [2]. These regulatory systems are typically composed of an inner membrane-bound sensor kinase (SK) and a cytoplasmic response regulator (RR). Environmental signals are often sensed by a periplasmic region of the SK, which then undergoes autophosphorylation followed by transfer of the phosphate to the RR. RR phosphorylation enhances DNA binding to recognition sites located in the promoters of regulated genes, subsequently activating or repressing transcription.

We recently described a novel Salmonella two-component system (TCS), PreA/PreB [3], which is similar to the quorum-sensing regulatory system $\mathrm{Qse} B / \mathrm{QseC}$ in enterohemorrhagic Escherichia coli [4]. PreB is a membrane-bound SK, with a periplasmic region containing a putative iron binding site (DxxE), while PreA is an OmpR-class RR. The pre $A B$ locus was identified in a transposon mutagenesis screen for regulators of $p m r C A B$, a locus encoding a separate TCS required for resistance to polymyxin $B$ and itself part of the large PhoP/PhoQ TCS regulon. PreA activates by two-fold the transcription of $p m r C A B$ in a PhoP- and PmrA- response regulator-independent fashion.

The signals controlling the PreA/PreB TCS are not known, and genetic evidence suggests that during growth in rich media, PreB primarily functions as a protein phosphatase inhibiting PreA function [3]. Curiously, the increase in pmrCAB transcription caused by PreA/PreB does not lead to observable transcriptional activation of most of the PmrA/PmrB regulon, with the exception of $y i b D$, a putative glycosylase, nor does it lead to the alteration of the polymyxin resistance measured by MIC or time-to-death assays. Besides $p m r C A B$ and $y i b D$, no other targets of PreA/ PreB are known [3], but the relatedness of Salmonella PreA/PreB to E. coli QseB/QseC suggested a potential wider role for this TCS.

The E. coli QseB/QseC TCS has been shown in various reports to sense quorum signal AI-3 as well as the eukary- otic hormones epinephrine/norepinephrine [5]. Activation of QseB/QseC results in the induction of flagellar gene synthesis and motility. Recently, while examining this TCS in Salmonella Typhimurium, bacterial motility was shown to increase in response to norepinephrine in the presence of iron [6]. Furthermore, qseC mutants were shown to possess virulence defects in rabbits (E. coli mutants) and pigs (S. Typhimurium mutants) $[5,6]$.

In this work, we describe the use of DNA microarrays to explore the genome-wide transcriptional effects of nonpolar mutations in preA/preB or of overexpression of the preA response regulator. These arrays corroborate previously published work relating to the role of PreB in regulated gene expression, identify several predicted PreA/ PreB-regulated genes (many of which are located near preAB) and examine the role of this TCS in Salmonella pathogenesis.

\section{Methods}

\section{Bacterial strains and media}

E. coli and $S$. Typhimurium strains and plasmids used in this study are listed in Table 1[7-9]. Luria-Bertani (LB) broth and agar were used for strain maintenance, as well as cloning and expression experiments. When appropriate, antibiotics were added at the following concentrations: ampicillin, $100 \mu \mathrm{g} / \mathrm{ml}$; kanamycin, $25 \mu \mathrm{g} / \mathrm{ml}$; tetracycline, $15 \mu \mathrm{g} / \mathrm{ml}$.

\section{Standard molecular biology and genetic techniques}

DNA purification, molecular cloning, and PCR were performed following standard procedures [10]. Plasmids were mobilized by electroporation. Marked mutations were transferred between $S$. Typhimurium strains by P22 HT105 int-102 mediated generalized transduction as previously described [11]. Deletions were created previously using the lambda-Red procedure of Datsenko and Wanner $[3,12]$. DNA sequencing was performed using a Big Dye fluorescent terminator and an ABI3770 capillary sequencer at the Plant Microbe Genomic Facility (The Ohio State University).

\section{Microarray fabrication}

Details of the construction of the backbone version of the Salmonella array were described previously [13]. PCR products were purified using the MultiScreen PCR 96-well Filtration System (Millipore, Bedford, MA), and eluted in $30 \mu \mathrm{l}$ of sterile water. Subsequently, the products were dried, resuspended in $15 \mu \mathrm{l} 50 \% \mathrm{DMSO}$, and $5 \mu \mathrm{l}$ were rearrayed into 384-well plates for printing.

\section{Preparation of CDNA probes}

A $0.5 \mathrm{ml}$ overnight culture of $S$. Typhimurium was used to inoculate $10 \mathrm{ml}$ of $\mathrm{LB}$ and grown at $37^{\circ} \mathrm{C}$, with shaking to an $\mathrm{OD}_{600}$ of 0.6-0.7. When inducing ectopically expressed 
Table I: Bacterial strains, plasmids and primers

\begin{tabular}{|c|c|c|}
\hline Strains/Plasmids/Primers & Description & Source \\
\hline \multicolumn{3}{|l|}{ E. coli } \\
\hline $\mathrm{DH} 5 \alpha$ & supE44 $\Delta$ (lacZYA-argF) UI69 ( $\triangle 80$ lacZ $\Delta M / 5)$ hsdRI 7 recA endAI gyrA96 thi-I relA I & Gibco \\
\hline \multicolumn{3}{|l|}{ Salmonella enterica serovar Typhimurium } \\
\hline JSG2 10 & ATCC I4208 (CDC65 I6-60), wild type & ATCC \\
\hline JSGI998 & JSG210 0 preA1998 & [3] \\
\hline JSG2343 & JSG2I $0 \Delta$ preB2343 & [3] \\
\hline JSG2626 & JSG210 $\triangle$ preAB2626 & [3] \\
\hline JSGI 225 & fliA::Tn / OdTet & gift of K. Klose \\
\hline JSG648 & phoN::cam prgHI::TnphoA & [7] \\
\hline \multicolumn{3}{|l|}{ Plasmids } \\
\hline$P_{B A D} 18$ & ColEI ori, pBAD L-Ara inducible $\left(A p^{r}\right)$ & [9] \\
\hline PRK2013::Tn7 & ColEI mob $\Delta$ traRK2 $\Delta$ repRK2 repE kan::Tn7 $\left(\mathrm{Tp}^{r} \mathrm{Sm}^{r} \mathrm{Sp}{ }^{r}\right)$ & [8] \\
\hline PJSG2558 & $\mathrm{P}_{\mathrm{BAD}} 18$ with a $0.7-\mathrm{kb}$ fragment containing preA expressed from pBAD $\left(A \mathrm{p}^{\mathrm{r}}\right)$ & [3] \\
\hline PJSG258I & $P_{B A D} 18$ with a $1.5-k b$ fragment containing preAB expressed from pBAD (Apr) & [3] \\
\hline \multicolumn{3}{|c|}{ 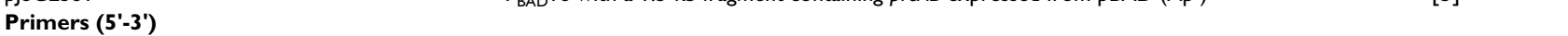 } \\
\hline 6-FAM-ccatcgccaataagtgtgtc & preA Reverse (primer ext.) & This study \\
\hline 6-FAM-cagggtgtcattcaactggc & mdaB Reverse (primer ext.) & This study \\
\hline 6-FAM-gatgacgctcaatgtggtcg & STM3I75 Reverse (primer ext.) & This study \\
\hline 6-FAM-ttcgcaaactggtcgaggac & ygiN Reverse (primer ext.) & This study \\
\hline 6-FAM-tgatcacgtacatggagtag & parC Reverse (primer ext.) & This study \\
\hline 6-FAM-gtagaacacagtgccataac & ygiW Reverse (primer ext.) & This study \\
\hline ggtagaacacagtgccataac & preA F (primer ext.) & This study \\
\hline ctcggtaaaccagtcgacgc & preA R (primer ext.) & This study \\
\hline ggaaggtggatttgaggc & mdaB F (primer ext.) & This study \\
\hline gcagcttcaccgtcagagata & mdaB F (primer ext.) & This study \\
\hline gacgatcttaacctgatgacc & mdaB R (primer ext.) & This study \\
\hline cgaagtggataaagactggaac & STM3I 75 F (primer ext.) & This study \\
\hline tagcgatagagcggaagc & STM3I75 R (primer ext.) & This study \\
\hline gcgtctatctgccattcc & ygiN F (primer ext.) & This study \\
\hline gcggcatgatccaccatc & ygiN R (primer ext.) & This study \\
\hline cctgaatttcgtccatgagg & parC F (primer ext.) & This study \\
\hline gaatagcgagattcctggcg & parC F (primer ext) & This study \\
\hline ccagctctgacatcgcatag & parC R (primer ext.) & This study \\
\hline ccatcgccaataagtgtgtc & ygiW F (primer ext.) & This study \\
\hline cgtcacgcagcgatttagc & ygiW R (primer ext.) & This study \\
\hline ggccgaacactctttgtggt & dnaN F (real-time) & This study \\
\hline gtataatttcggtcgcatccgt & dnaN R (real-time) & This study \\
\hline atatcgtcgagcgcatttcc & ygiW F (real-time) & This study \\
\hline tccagtctttatccacttcgcc & ygiW R (real-time) & This study \\
\hline aagagttcgcgttgctggaa (JG | | 34) & preA F (real-time, RT-PCR) & This study \\
\hline gagcttgcggcgtaaatgat & preA R (real-time) & This study \\
\hline agactctggcgcctgactcg & ygiN F (real-time) & This study \\
\hline aacgccggattccagaatacg & ygiN R (real-time) & This study \\
\hline acaggcttaagagtagcggctg (JG| |37) & preB R (RT-PCR) & This study \\
\hline atatcgtcgagcgcatttcc (JG|| 32) & ygiW F (RT-PCR) & This study \\
\hline cgcggatccttaacgaagcggcagatagatatc (JG I 223) & STM 3175 R(RT-PCR) & This study \\
\hline gtgtcgtttggcaacgccgcggaa (JGI703) & preB F(RT-PCR) & This study \\
\hline caactggccgttggagtgcgcg (JGI704) & $m d a B$ R (RT-PCR) & This study \\
\hline tgccggatgttccgcgctataccgca (JGI705) & $m d a B F(R T-P C R)$ & This study \\
\hline tgacggtgatgttggcccggacgcg (JG |706) & ygiN R (RT-PCR) & This study \\
\hline gaagccgtccagcagttg (JG|86I) & STM I595 F (Real-time PCR) & This study \\
\hline gcgataaccattccaccaaac (JG I 862) & STM I595 R (Real-time PCR) & This study \\
\hline cgttcctaaacttgcgttacag (JG I863) & STM 3 I 75 F (Real-time PCR) & This study \\
\hline gctggcgttgaccttatcc (JG | 864) & STM 3175 R (Real-time PCR) & This study \\
\hline ttgtatctggagattgtggactac (JG|865) & STM I 685 F (Real-time PCR) & This study \\
\hline gagcccgtcgcaaagttg (JG|866) & STM I685 R (Real-time PCR) & This study \\
\hline tctacgcttgttcgcttac (JG | 867) & STM I252 F (Real-time PCR) & This study \\
\hline ggtgttgtccagatattatgttc (JG I868) & STM I 252 R (Real-time PCR) & This study \\
\hline tacagtggacaatgaatg $(\mathrm{G} \mid 869)$ & STM I 684 F (Real-time PCR) & This study \\
\hline gctatggctatgtaacag (JGI870) & STM I 684 R (Real-time PCR) & This study \\
\hline ggcttcacggcggcaatg (JGI87I) & STM 2080 F (Real-time PCR) & This study \\
\hline tcacgatacgggagggataaagg (JG|872) & STM 2080 R (Real-time PCR) & This study \\
\hline ctaacttccaggaccactc $(\mathrm{G} \mid 873)$ & STM 4I I 8 F (Real-time PCR) & This study \\
\hline gataaccgtacagactcatac (JG I 874) & STM 4I I8 R (Real-time PCR) & This study \\
\hline tgatatgggcgttctggtctg (JG I875) & STM I 253 F (Real-time PCR) & This study \\
\hline cgtgctgccagtgaggag (JG|876) & STM I 253 R (Real-time PCR) & This study \\
\hline
\end{tabular}


preA (or vector controls) with $10 \mathrm{mM}$ arabinose, medium was buffered with $100 \mathrm{mM}$ TrisHCl. Samples were transferred into chilled Falcon tubes containing $2 \mathrm{ml}$ of $5 \%$ phenol/95\% ethanol, incubated 15 min on ice, and cells were collected by centrifugation at $8000 \mathrm{~g}$ for $10 \mathrm{~min}$ at $4{ }^{\circ} \mathrm{C}$. Cells were lysed and RNA was collected, purified and DNase treated according to Promega SV Total RNA Isolation Kit (Promega, Madison, WI). RNA was checked for quantity and quality via gel electrophoresis or the Experion System (Bio-Rad, Hercules, CA). Cy3- and Cy5-dyelinked dUTP was directly incorporated during reverse transcription from total RNA to synthesize labeled cDNA probes, based on the method described by [13] with the following modifications: $15-100 \mu \mathrm{g}$ of total RNA and 2.4 $\mu \mathrm{g}$ of random hexamers were resuspended in $30 \mu \mathrm{l}$ of water, and subsequently the amounts and volumes of all components were doubled. Furthermore, $2 \mu$ l of RNase inhibitor (Invitrogen, Carlsbad, CA) was added to the reverse transcription, and the reaction incubated at $42^{\circ} \mathrm{C}$ for $2 \mathrm{~h}$. After the first hour of incubation, $2 \mu \mathrm{l}$ of additional Superscript II reverse transcriptase was added. Probes were purified using the QIAquick PCR purification kit (Qiagen, Valencia, CA) and eluted in $50 \mu \mathrm{l}$ sterile water. Subsequently, probes were dried down to $20 \mu \mathrm{l}$ final

volume.

\section{Hybridization and data acquisition}

Probes were mixed with equal volumes of $2 \times$ hybridization buffer containing 50\% formamide, 10× SSC and $0.2 \%$ SDS, and boiled for $5 \mathrm{~min}$. Probes were hybridized to the Salmonella array overnight at $42^{\circ} \mathrm{C}$ using a hybridization chamber (Corning, Corning, NY) submerged in water. Protocols suggested by the manufacturer for hybridizations in formamide buffer were applied for prehybridization, hybridization and post-hybridization wash processes. Scans were performed on an Affymetrix 428 Laser scanner (Affymetrix, Santa Clara, CA) using the Microarray suite 5.0 (Affymetrix) software.

\section{Data analysis}

The TIFF files where unstacked using ImageJ (NIH) and signal intensities were quantified using the QuantArray 3.0 software package (Packard BioChip Technologies, Billerica, MA) at the Sidney Kimmel Cancer Center of San Diego. Unless noted otherwise, at least two slides (each containing triplicate arrays) were hybridized reciprocally to Cy3- and Cy5-labeled probes per experiment. Spots were analyzed by adaptive quantitation, and local background was subsequently subtracted from the recorded spot intensities. Ratios of the contribution of each spot to total signal in each channel were calculated (data normalization). Negative values (i.e., local background intensities higher than spot signal) were considered no data. The median of the six ratios per gene was recorded. For cDNA probes, ratios and standard deviations were calculated between the two conditions (e.g., experiment versus control). Genes with signals less than two standard deviations above background in both conditions were considered as not detected. The microarray data can be found at Gene Expression Omnibus http://www.ncbi.nlm.nih.gov/geo/ under series number GSE12866.

\section{Real time quantitative RT-PCR (qRT-PCR)}

Two micrograms of RNA purified with the same protocol utilized for microarray analysis (but on different dates from different cultures) was used to synthesize cDNA using Invitrogen Superscript II in $25 \mu$ l reactions. Quantitative analysis of cDNAs and Ct value estimation was performed with an iCycler iQ5 system using SYBR Green I DNA binding dye (BioRad, Hercules, CA) to detect PCR products. The PCR mixture was prepared by mixing 12.5 $\mu \mathrm{l}$ 2X iQ SYBR Green, $0.5 \mu \mathrm{M}$ of each primer (Table 1), and $50 \mathrm{ng}$ of cDNA template. Parameters for the amplification were: initial denaturation at $95^{\circ} \mathrm{C}$ for $10 \mathrm{~min}$, followed by 40 cycles each consisting of $15 \mathrm{~s}$ at $95^{\circ} \mathrm{C}, 30 \mathrm{~s}$ annealing at $55^{\circ} \mathrm{C}$. The efficiency of amplification for each target gene was evaluated by calculating standard curves generated from 10-fold dilutions of each template sample followed by estimation using the regression model $(\mathrm{Ct}=\mathrm{m} \times \log ($ Dilution $)+\mathrm{b})$. In all cases the efficiency ranged from 95 to $100 \%$. Relative fold differences of gene expression between treatments were calculated using the $2^{-\Delta \Delta \mathrm{Ct}}$ method with $16 \mathrm{~S}$ rRNA or $\operatorname{dnaN}$ as standards. All qRT-PCR experiments were performed in triplicate at least twice with similar results.

\section{Operon transcript mapping by RT-PCR}

Primers within the orfs for preA, preB, mdaB, ygiN, ygiW, and STM3175 were designed and used in RT-PCR reactions to determine if genes were co-transcribed. RNA from OD 0.6 cultures was isolated and cDNA was produced as described above. All RT-PCR experiments were performed on two separate occasions with cDNA derived from separate RNA preparations, each with similar results.

\section{Primer extension}

Analysis of the 5' ends of mRNA transcripts was performed by primer extension as described by Merighi et al. 2006 [3]. 6-FAM-labeled primers (Table 1) and $50 \mu \mathrm{g}$ cDNA were analyzed in an ABI 3770 capillary electrophoresis sequencer at the Plant Microbe Genomic Facility (The Ohio State University) along with DNA sequencing reactions using the same primer. In particular, Thermoscript was substituted for Superscript II (Invitrogen, Carlsbad, CA) and higher extension temperatures $\left(65^{\circ} \mathrm{C}\right)$ were used to obviate secondary structure problems. All extension reactions were performed at least twice with independent RNA preparations and the reproducible peaks were selected. 


\section{Animal cell cultures and invasion assay}

HeLa cell lines were obtained from ATCC (Manassas, VA). Cells were grown to a monolayer at $37^{\circ} \mathrm{C}, 5 \% \mathrm{CO}_{2}$ in DMEM with $10 \%$ heat-inactivated fetal bovine serum. Cells were then infected at an MOI of 100 in 24-well plates. Bacteria were spun onto the HeLa cells and incubated at $4^{\circ} \mathrm{C}$ for $30 \mathrm{~min}$, then at $37^{\circ} \mathrm{C}$ for 1 hour. Extracellular bacteria were killed with $50 \mu \mathrm{g} / \mathrm{ml}$ gentamicin for 30 min. HeLa cells were then lysed with $0.1 \%$ Triton X100 and plated for CFU determination.

\section{Mouse studies}

Food and water were withdrawn $4 \mathrm{~h}$ before inoculation of female BALB/c mice (weighing 16 to $18 \mathrm{~g}$ ). Mice (10 for each strain) were inoculated with $10^{6}$ bacteria by oral gavage using a 22-gauge feeding needle. Dilutions of the stationary-phase cultures were plated to determine the number of bacteria present in the inoculum. For virulence assays, time of death was recorded as days post-infection. Competition infection experiments were conducted as described above, except that the mutant strain was coinfected with a chloramphenicol marked wild type strain (JSG224, phoN2 ZXX::6251dTn10-Cam). After plating bacteria on appropriate media from organs four days post-infection, the competitive index was calculated as the

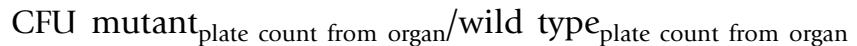

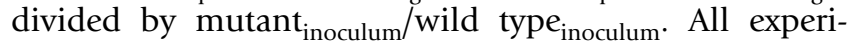
ments were reviewed and approved by the Ohio State University Institutional Animal Care and Use Committee.

\section{Motility assays}

$0.3 \%$ agar DMEM plates were made containing, where indicated, 10 or $20 \mu \mathrm{M}$ autoinducer-2 (AI-2 was a gift from Dr. Dehua Pei, Department of Chemistry, The Ohio State University), 10 or $50 \mu \mathrm{M}$ epinephrine, or equivalent amounts of acidified water as a control for epinephrine plates (epinephrine was solubilized in acidified water). Overnight cultures were grown in $\mathrm{LB}, 37^{\circ} \mathrm{C}$ with shaking, adjusted to an OD of 0.1 at $600 \mathrm{~nm}$ and incubated for 2 hours at $37^{\circ} \mathrm{C}$ with shaking. Plates were stab-inoculated and incubated at $37^{\circ} \mathrm{C}$ for 14 hours. The diameter of the motility circles were measured at various times and compared.

\section{Results}

Transcriptome of the PreA/PreB two-component system In previous experiments, we realized that the pre $A B$ TCS was not fully activated during growth in LB, as indicated by the absence of regulatory effects on the two known target genes ( $y i b D, p m r C A B$ ) when comparing a nonpolar mutation in the preA response regulator to the wild type strain [3]. This was confirmed in this study by microarray analysis co-hybridizing preA and wild type cDNA to a multistrain slide microarray of Salmonella enterica (data not shown). In our previous studies, transcriptional changes of the pre $A B$ target genes upon growth in $\mathrm{LB}$ was achieved by overexpression of PreA or by mutation of the sensor kinase preB [3]. We therefore analyzed the effect of overepressing PreA in a $\triangle p r e A$ strain carrying preA driven by a $\mathrm{PBAD}$ arabinose-inducible promoter grown in buffered LB. In addition, past experiments had implied that PreB may be acting as a protein phosphatase when bacteria are grown in LB [3]. If this is the case, some of the regulatory effects attributed to preA may have been dampened in the previous experimental design. We therefore proceeded to also analyze the cDNA from a pre $A B$ double mutant expressing pBAD-preA and a pre $A B$ strain carrying the vector control. All of the data from both experiments is included in Additional file 1, but a focused list of key candidate regulated genes is shown in Table 2 .

Many of the genes upregulated in the $\Delta p r e A$ strain overexpressing preA (Table 2, column 1) were reconfirmed in experiments with the pre $A B$ mutant strain overexpressing preA (Table 2, column 2), but with increased fold activation. For instance, yibD was upregulated $\sim 69$-fold in the pre $A B$ mutant with $\mathrm{pBAD}$-preA compared to the $\sim 2$-fold seen in PreB ${ }^{+}$backgrounds, while $m d a B$ was upregulated $\sim 7$-fold versus 2 -fold in the PreB $^{+}$background. In addition, one of the discernable patterns from the two microarrays was that the three genes flanking the preAB operon: ygiW, STM3175, mdaB, were upregulated 37-, 21-, and $~ 7$ fold, respectively (Table 2, column 2). Furthermore, in the pre $A B$ mutant background, we also observed upregulation of additional genes belonging to the PhoP/PhoQ and PmrA/PmrB regulons: pmrAB, udg, cptA (STM4118) and $p a g P$. This further supports the connection between pre $A B$ and the two major regulons controlling genes involved in LPS modifications and antimicrobial peptide resistance in Salmonella and provides confidence to the quality of our microarray experiments.

\section{qRT-PCR analysis and transcriptional organization of preAB and flanking genes}

To confirm the results of the microarray and to examine the regulation of pre $A B$ and the genes surrounding it, we performed qRT-PCR. The preA gene was shown to be induced 344 -fold in a $\triangle p r e B$ strain vs. a wild type strain, furthering the previous finding of PreB acting primarily as a phosphatase when grown in LB and providing evidence of PreA-mediated positive autoregulation of preAB. The induction of preB in the microarray of the preA mutant background overexpressing preA also provided evidence of positive autoregulation of preAB (supplement Table 1). ygiW was strongly activated by PreA (355-fold) when comparing expression in a $\triangle$ preAB/pBAD18-pre $A^{+}$strain vs. $\triangle p r e A B / p B A D 18$. Using these same strains, $y g i N$ was more weakly activated by PreA (2.94-fold). Several other PreA-regulated genes including STM3175 (605.3-fold) and $m d a B$ (32.5-fold) were also analyzed by qRT-PCR, all 

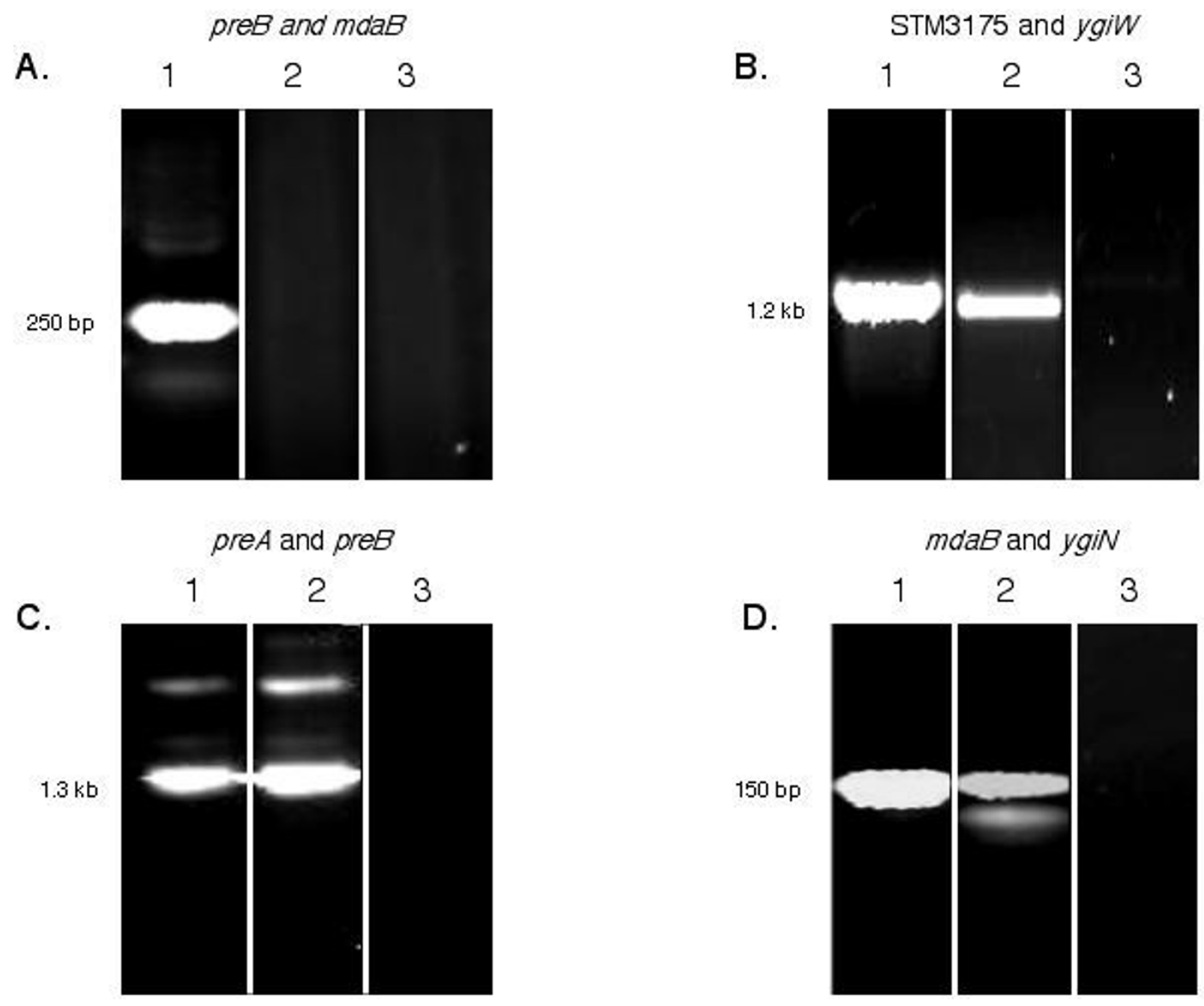

E.
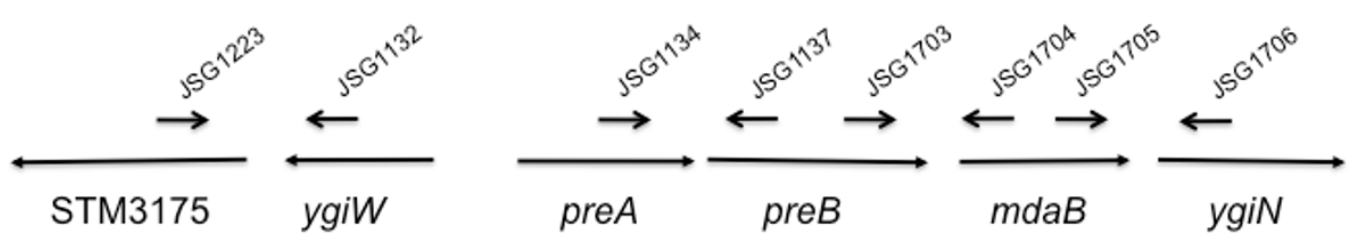

Figure I

Co-transcription analysis of the genes in the local chromosomal region surrounding preA. (A-D) The sets of genes examined are described above the ethidium bromide stained gels. The lane assignments in each set: (I) chromosomal DNA as a template; (2) cDNA as a template; (3) cDNA as a template, no reverse transcriptase. (E) A graphic representation of the preA-linked genes and the primers used for RT-PCR. The sequences for the primers can be found in Table I. 
Table 2: Microarray and real time PCR analysis showing a limited list of genes ${ }^{a}$ predicted to be PreAB activated

\begin{tabular}{|c|c|c|c|c|c|}
\hline ORF & Gene & Function & $\begin{array}{l}\text { Microarray } A^{b} \\
M^{d} \text { (fold change) }\end{array}$ & $\begin{array}{l}\text { Microarray } B^{c} \\
M \text { (fold change) }\end{array}$ & qRT-PCRe \\
\hline STM3707 & yibD & putative glycosyltransferase & $0.8(1.7)$ & $6.1(68.6)$ & NPf \\
\hline STM3। 76 & ygiW & Membrane protein (DUF388; exporter?) & $4.5(22.6)$ & $5.2(36.8)$ & 355 \\
\hline STMI 253 & & Cytochrome b56I ( $\mathrm{Ni}^{2+}$ dependent $)$ & $2.9(7.5)$ & $4.9(29.9)$ & 372 \\
\hline STMI595 & $\operatorname{srfC}$ & ssrAB activated gene; predicted coiled-coil structure & $4.3(19.7)$ & $4.7(26.0)$ & 1.2 \\
\hline STM3।75 & & putative bacterial regulatory helix-turn-helix proteins, $\mathrm{AraC}$ family & $3.6(12.1)$ & $4.4(21.1)$ & 605.3 \\
\hline STMI 685 & $y c j X$ & putative ATPase & $2.3(4.9)$ & $3.8(13.9)$ & 37.7 \\
\hline STMI 252 & & putative cytoplasmic protein & $1.5(2.8)$ & $2.8(7.0)$ & 8.6 \\
\hline STM3I79 & $m d a B$ & NADPH specific quinone oxidoreductase (drug modulator) & $1.0(2.0)$ & $2.8(7.0)$ & 32.5 \\
\hline STMI 684 & ycjF & putative inner membrane protein & I.I (2.I) & $2.6(6.1)$ & 61.2 \\
\hline STM429I & pmrB & sensory kinase in two-component regulatory system with PmrA & NDg & $2.1(4.3)$ & NP \\
\hline STM2080 & udg & UDP-glucose/GDP-mannose dehydrogenase & ND & $1.8(3.5)$ & 23.2 \\
\hline STM4292 & pmrA & response regulator in two-component regulatory system with $\mathrm{PmrB}$ & ND & $1.7(3.2)$ & NP \\
\hline STM4II8 & yijP $(c p t A)$ & putative integral membrane protein & ND & I.5 (2.8) & 32.8 \\
\hline STM0628 & pagP & PhoP-activated gene, palmitoyl transferase & ND & I.I (2.I) & NP \\
\hline STM2238 & & putative phage protein & $0.9(1.9)$ & $1.0(2.0)$ & NP \\
\hline
\end{tabular}

a This list includes only those genes that were upregulated in both the preA and preAB mutant strains overexpressing preA, those confirmed by realtime PCR, genes previously shown to be preA-regulated (yibD, pmrAB) or those known to belong to the PhoPQ or PmrAB regulons

b $\triangle$ preA/pBADI 8-preA vs. $\triangle$ preA/pBADI8

c $\triangle$ preAB/pBADI8-preA vs. $\triangle$ preAB/pBADI8

$\mathrm{d} M=\log _{2}$ (expression plasmid/vector control)

e real time PCR ( $\mathrm{QRT}-\mathrm{PCR}$ ) performed with cDNA derived from the strains used in Microarray $B$

${ }_{\mathrm{f}} \mathrm{NP}=$ not performed

gND $=$ not detected

confirming the regulation observed in the microarrays (though not always matching the observed fold-change) (Table 2).

The transcriptional organization of the preAB operon and of the genes flanking it, which were strongly upregulated by PreA, were analyzed by RT-PCR. As shown in Fig. 1, PCR fragments spanning preA and preB, ygiW and STM3175, and $m d a B$ and ygiN were observed, suggesting that these sets of genes are co-transcribed. While primers spanning preB and $m d a B$ (separated by a 106 bp intergenic region) yielded PCR product using a DNA template, no such product was observed with cDNA, even with the use of multiple primer sets, suggesting that these genes are not co-transcribed. These data, coupled with the microarray results, suggest that PreA is necessary for the activation of the $y g i W$-STM3175, preA-preB, and mdaB-ygiN operons.

We next attempted to map the transcriptional start sites of these three operons by primer extension using a fluorescent primer protocol. Using this approach, the start of transcription for the pre $A B$ operon was identified at -423 / $424 \mathrm{bp}$ from the start codon, implying that the preAB promoter is internal to $y g i W$ and contains a large, untranslated leader region (Fig. 2). The start site of the ygiWSTM3175 operon was at $-161 \mathrm{bp}$, which is $10 \mathrm{bp}$ internal to the preA open reading frame. Multiple attempts were made to map the mdaB-ygiN start, however we were unsuccessful at identifying a clear site for transcriptional initiation.

\section{Phenotypes of preAB TCS mutants}

We previously reported that PreA/PreB is orthologous to the E. coli QseBC system, which responds to AI-3 and epinephrine/norepinephrine signals. In response to these signals, the QseC sensor kinase has been reported to affect motility in both $E$. coli and $S$. Typhimurium $[6,14]$. However, our microarray data did not suggest any major and/ or consistent effect of PreA/PreB on transcription of the flagellar operon. Therefore, we assessed the effects of mutations in preA and preB on the motility of $S$. Typhimurium on agar plates with DMEM as the culture medium. The results showed a reduction in motility for the preB sensor mutant (Fig. 3) but not for the preA or pre $A B$ mutants. As seen with QseC in E. coli, the addition of synthetic AI-2 did not complement the preB mutant motility defect and also did not affect the motility of the wild type strain (Fig. 3A). Additionally, though epinephrine/norepinephrine has been reported to activate motility in both E. coli and $S$. Typhimurium $[6,15]$, a slight but non-significant increase in wild type strain motility was observed in our assays using identical conditions and epinephrine concentrations used previously in E. coli. Supplementation of the media with epinephrine did increase the motility of preA, preB and pre $A B$ mutants (all statistically significant except preB, Fig. 3B), but as this effect of epinephrine on $S$. Typhimurium motility was observed only in 
A.

B.
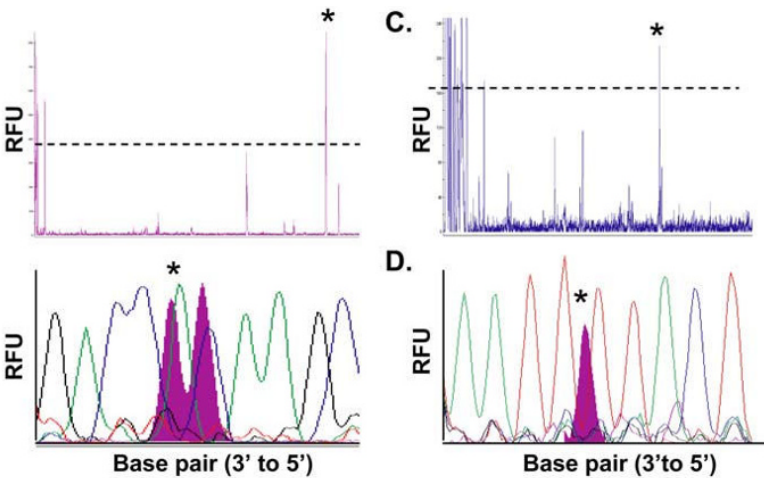

D.

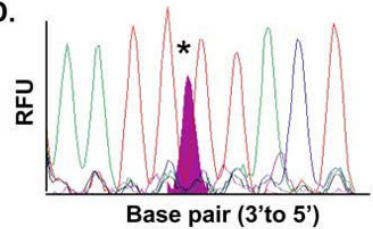

E.

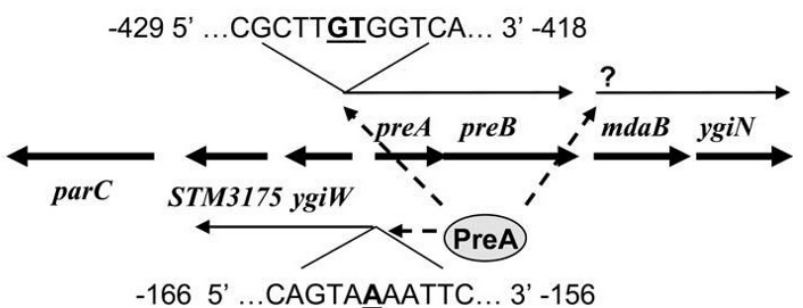

Figure 2

Fluorescent primer extension analysis of transcriptional start sites for the preAB and ygiW-STM3 I 75 operons. Electropherograms of the labeled cDNA are shown for preA $(A)$ and ygiW $(C)$. Dashed lines mark the relative fluorescence unit (RFU) cut-off, below which does not give a confident signal strength. Asterisks (*) denote which cDNA peak was analyzed. Labeled cDNA electropherograms (filled peaks) were aligned with sequence chromatograms (open peaks) to identify the base at which transcription starts for both preAB (B) and ygiW-STM3 I75 (D). Results of transcriptional organization are diagramed as shown with start sites mapped relative to the translational start (E). PreA appears to activate transcription of each of the three operons defined in the preA region (dashed lines denote positive regulation).

preA or preB mutant strains, this effect is not mediated by PreA/PreB.

Overexpression of $m d a B$ [16] and mutation of preB ( $y g i Y$; [17]) were previously shown to affect drug resistance in $E$. coli and oxidative stress response in Helicobacter spp. [1820]. In addition, catalase genes appear PreA-regulated (Additional file 1). pre $A B$ mutant strains were therefore analyzed for resistance to various chemicals and antibiotics, including nalidixic acid, pyrazinoic acid, $\mathrm{H}_{2} \mathrm{O}_{2}$, paraquat, adriamycin, and tetracycline. None of the mutants showed increased sensitivity when compared to the wild type strain (data not shown).

To determine if the PreA/PreB system affects virulence, mutant and wild type strains were perorally inoculated in
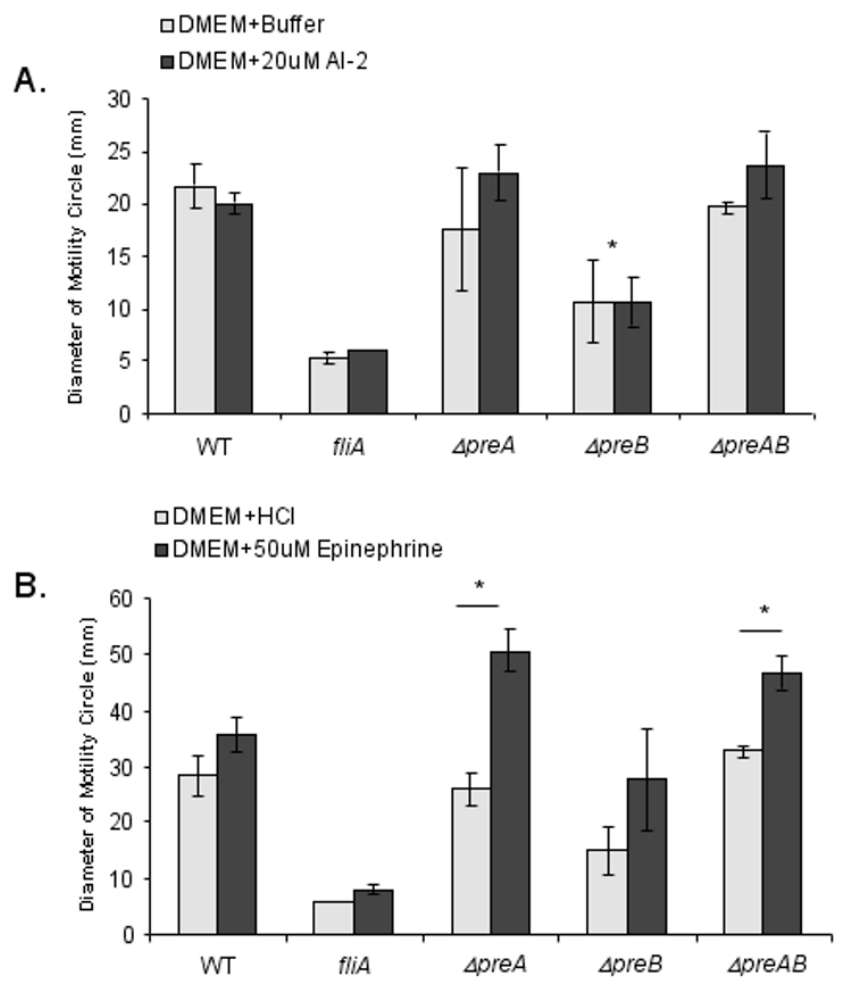

Figure 3

Motility assays were performed using DMEM soft agar plates. Motility ring diameters of the wild type I4028s strain and a negative control (fliA) were compared to preA, pre $B$, and pre $A B$ strains. Signaling molecules were tested for possible affects on motility. (A) $20 \mu \mathrm{M} \mathrm{Al-2} \mathrm{(dark} \mathrm{bars)} \mathrm{or} \mathrm{an}$ equal volume of buffer (light bars) were added to the medium. (B) $50 \mu \mathrm{M}$ epinephrine (dissolved in acidified water, dark bars) or an equal volume of acidified water (light bars) was added to medium. An asterisk (*) denotes statistical significance with a $p$-value $<0.02$ as determined with a student t-test. The asterisk in $(\mathrm{A})$ is in comparision of $\Delta p r e B$ to the wild type strain.

mice and mortality was recorded over two weeks. The preA mutant showed no virulence defect while mice infected with the pre $A B$ strain showed a consistent two day delay in mortality, but eventually all mice succumbed to infection (Fig. 4). The pre $A B$ mutant strain also demonstrated a consistent competition infection defect (competitive index: spleen, 0.344; liver, 0.326) when co-inoculated by oral gavage with the wild type strain, which was not observed with strains containing single mutations in pre $A$ or pre $B$ (data not shown). Thus, the PreA/PreB TCS has a slight but reproducible effect on virulence in mice.

Given that invasion of the small intestine is a prerequisite to systemic infection upon oral inoculation, we also evaluated the ability of various preA/preB mutants to invade HeLa cells grown in vitro. Again, the response regulator 


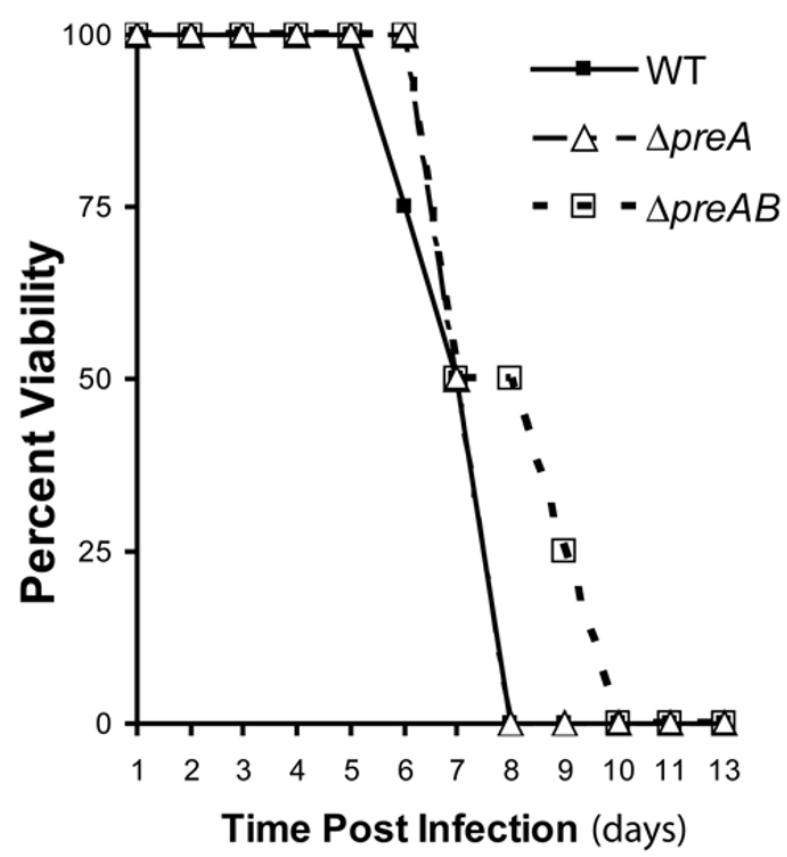

Figure 4

Female BALB/c mice were inoculated with $10^{6}$ bacteria via oral gavage and animals were monitored over a period of 13 days.

(preA) mutant did not show any defect in invasion of HeLa cells. The preB strain showed a marginal and nonsignificant reduction in invasion upon 2 hours co-cultivation at a $\mathrm{MOI}=100$ (invasion $\sim 80 \%$ of wild type), while a larger defect was observed for the pre $A B$ double mutant ( 30\% of WT) (Fig. 5). Therefore, the PreA/PreB TCS has a direct or indirect effect on host cell invasion.

\section{Discussion}

TCSs are important for bacterial survival in host and nonhost conditions. We previously identified a TCS (PreA/ PreB/QseB/QseC) that indirectly affected the transcriptional activation of the PmrA/PmrB TCS of Salmonella [3]. Some of the genes of the PmrA/PmrB regulon were affected by PreA/PreB, but antimicrobial peptide resistance mediated by PmrA/PmrB was unaffected by the presence of PreA/PreB. Because we had few clues to the potential function of this TCS in Salmonella, we pursued a microarray approach to identify regulated genes that might suggest phenotypes related to PreA/PreB.

Previous research demonstrated that PreB acts preferentially in laboratory growth media (e.g. LB) in a negative manner with regard to PreA gene regulation- likely acting as a phosphatase leaving PreA unphosphorylated and

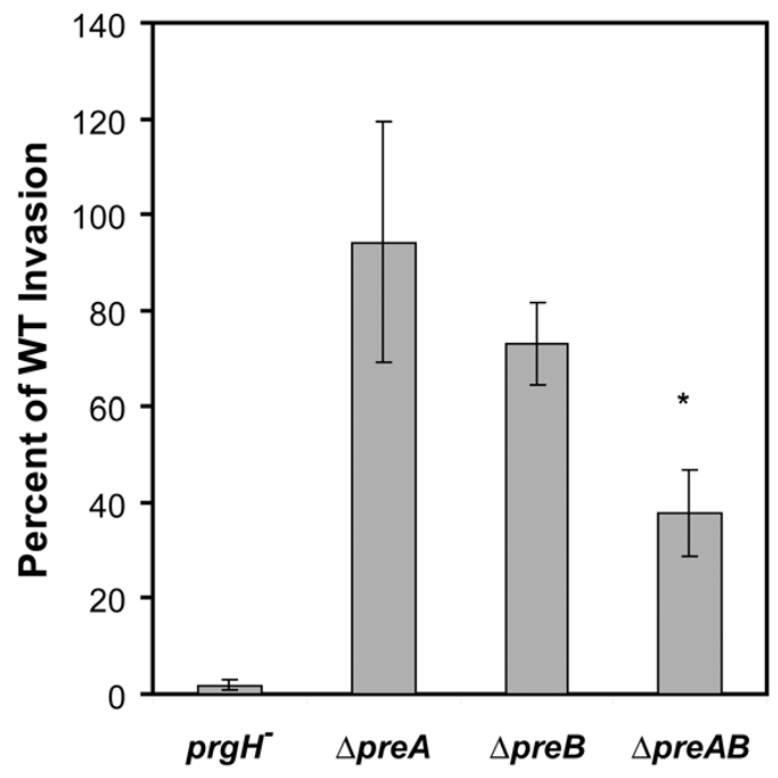

Figure 5

HeLa cell invasion assays were performed for wild type, prgH (negative control), preA, pre $B$, and preAB strains. HeLa cells were grown to monolayer in DMEM with $10 \% \mathrm{FBS}$ at $37^{\circ} \mathrm{C}$ and $5 \% \mathrm{CO}_{2}$. Cells were then infected with bacteria at an $\mathrm{MOI}$ of 100 in 24-well plates. Data is presented as percent of wild type CFUs. The asterisk (*) denotes statistical significance with a $P$-value of $<0.02$ as determined with a student t-test.

inactive. We have not yet identified a growth condition where this is not the case. These observations also held true with the microarray analysis, as we observed more regulated genes and a higher level of regulation in the absence of PreB than in its presence. This was true even when PreA was overexpressed. Thus, in the absence of known environmental conditions that activate this TCS, the strain expressing the most PreA-regulated loci is one in which PreA is overexpressed in the absence of PreB.

Comparison of the results of two microarray analyses, (preA mutant/ppreA [PreA overexpressed] vs. preA mutant with empty vector; preAB mutant/ppreA [PreA overexpressed] vs. pre $A B$ mutant with empty vector), showed reasonable agreement, with about $40 \%$ of the genes in the pre $A$ mutant background array also observed in the pre $A B$ mutant background array (Additional file 1; Table 2). There were few candidate repressed loci but these were more numerous than the activated genes in the preAB mutant ppre $A$ vs. pre $A B$ mutant with empty vector arrays. If our model concerning the phosphatase function of PreB is accurate, this may suggest that phosphorylation of PreA is required for it to act as a repressor. 
The repressed and activated genes in the Additional file 1 and Table 2 show little commonality, except the presence of known PmrA-regulated genes [STM3707 (yibD), STM1252/53, STM4292 (pmrA), STM4291 (pmrB), STM2080 (ugd/pmrE), and STM4118 (yijP/cptA)] and genes in the local region around preA [STM3177 (preA), STM 3178 (preB; from Table 2), STM3176 (ygiW), STM 3175, and STM $3179(m d a B)]$. We further analyzed the transcriptional units located in the vicinity of preA, showing that the PreA- activated operons were composed of preA-preB, $m d a B-y g i N$, and $y g i W-S T M 3175$. preB and $m d a B$ were not shown by RT-PCR to be co-transcribed. The operonic arrangement of pre $A$ and preB and the activation of this operon by PreA are in agreement with the study of qseBC in enterohemorrhagic E. coli (EHEC) ([21]).

Transcriptional initiation sites were defined for preA-preB and $y g i W$-STM3175 but not mdaB-ygiN. The preAB start site does not match those mapped for qseBC in EHEC, which occur at -27 and -78 with respect to the qseB ATG. However, QseB binds to the EHEC qseBC promoter near its transcriptional starts $(-27$ to -40$)$ but also in a region (409 to -423 ) that is located near the transcriptional initiation site we mapped for preAB [21]. We hypothesize that PreA binds to the promoter region of each of these operons (preA-preB, mdaB-ygiN, and $y g i W$-STM3175) to activate transcription, and future work will define the PreA binding sites in these regulated promoters.

It has been previously demonstrated that QseC (PreB ortholog) of EHEC is a receptor for host-derived epinephrine/norepinephrine and intestinal flora derived AI-3 [5]. In E. coli, QseB positively regulates the transcription of flagellar genes and thus flagellar synthesis and motility. $S$. Typhimurium motility has also been shown to be affected by norepinephrine and QseC/PreB [6]. However, we were unable to demonstrate a role of PreA/PreB in the regulation of flagellar genes or a role for PreA/PreB in motility, except for an effect of a preB mutation alone. Furthermore, the addition of AI-2 or epinephrine had no effect on wild type motility. Epinephrine did surprisingly increase motility of pre $A$ and pre $A B$ mutants, but this effect was clearly PreA/PreB independent. Recently, Bearson et al. [22] demonstrated that norepinephrine acts as a siderophore, and that mutations affecting iron transport no longer responded to norepinephrine. Thus it remains a strong possibility that any effects observed on bacteria by epinephrine/norepinephrine are due to enhanced iron availability. PreB contains a putative iron binding motif in its periplasmic region, thus furthering a presumed association of iron with the regulation of PreA/PreB.

Though PreA/PreB regulates genes that affect antimicrobial peptide resistance ( $p m r A B, c p t A)$ and resistance to a variety of drugs $(m d a B)$ or reactive oxygen compounds (e.g. katE, STM1731, dps), none of the preA or preB mutations affected antimicrobial susceptibility. However, the loss of both preA and preB affected both invasion of epithelial cells in vitro (though no consistant effect of PreA/ $\mathrm{B}$ on Salmonella Pathogenicity island 1 invasion genes was observed) and virulence in the mouse model. Future work will focus on genes regulated by PreA/PreB that contribute to these phenotypes.

\section{Conclusion}

PreA/PreB is a TCS that regulates Salmonella genes including those of the PmrA/PmrB regulon and those adjacent to pre $A B$ on the chromosome. RNA analysis of the genes surrounding preA revealed three PreA-activated operons composed of preA-preB, mdaB-ygiN, and $y g i W$-STM3175. Though PreA/PreB do not appear to be responsive to hostderived hormones or microbial quorum-sensing signals as has been previously reported, PreA/PreB do play a role in Salmonella host cell invasion and virulence.

\section{Authors' contributions}

AB performed RT-PCR and other RNA experiments. AC-P perfomed the initial work with this TCS and constructed some of the mutant strains. SP and MMc constructed the arrays and performed the microarray statistical analysis. MMc aided in the final preparation of the manuscript. ANS and MM together perfomed microarray analysis and all other experiments, and jointly wrote the first draft of the manuscript. JSG participated in the writing of the manuscript, the interpretation of the data, and conceived the study. All authors read and approved the final version of the manuscript.

\section{Additional material}

\section{Additional file 1}

Candidate PreA-regulated genes identified by microarray analysis. This table is a complete list of candidate PreA-regulated genes identified by microarray analysis of RNA isolated from strains overexpressing preA (in preA [Microarray A] and preAB [Microarray B] mutant backgrounds)

Click here for file

[http://www.biomedcentral.com/content/supplementary/14712180-9-42-S1.doc]

\section{Acknowledgements}

This work was supported by grant Al04352I from the NIH to JSG. MMc was supported, in part by NIH grants Al034829, Al52237, and Al07397I.

\section{References}

I. Stoycheva MV, Murdjeva MA: Antimicrobial therapy of salmonelloses - current state and perspectives. Folia Med (Plovdiv) 2006, 48:5-10.

2. Beier D, Gross R: Regulation of bacterial virulence by twocomponent systems. Curr Opin Microbiol 2006, 9:|43-I52. 
3. Merighi M, Majerczak DR, Zianni M, Tessanne K, Coplin DL: Molecular characterization of Pantoea stewartii subsp. stewartii HrpY, a conserved response regulator of the Hrp type III secretion system, and its interaction with the hrpS promoter. J Bacteriol 2006, 188:5089-5100.

4. Sperandio V, Torres AG, Kaper JB: Quorum sensing Escherichia coli regulators $B$ and $C$ (QseBC): a novel two-component regulatory system involved in the regulation of flagella and motility by quorum sensing in E. coli. Mol Microbiol 2002, 43:809-82I.

5. Clarke MB, Hughes DT, Zhu C, Boedeker EC, Sperandio V: The QseC sensor kinase: a bacterial adrenergic receptor. Proc Natl Acad Sci USA 2006, 1 03: 10420-10425.

6. Bearson $\mathrm{BL}$, Bearson SM: The role of the QseC quorum-sensing sensor kinase in colonization and norepinephrine-enhanced motility of Salmonella enterica serovar Typhimurium. Microb Pathog 2008, 44:27I-278.

7. Behlau I, Miller SI: A PhoP-repressed gene promotes Salmonella typhimurium invasion of epithelial cells. J Bacteriol 1993, 1 75:4475-4484

8. Ditta G, Stanfield S, Corbin D, Helinski DR: Broad host range DNA cloning system for gram-negative bacteria: construction of a gene bank of Rhizobium meliloti. Proc Natl Acad Sci USA 1980, 77:7347-735I.

9. Guzman LM, Belin D, Carson MJ, Beckwith J: Tight regulation, modulation, and high-level expression by vectors containing the arabinose PBAD promoter. J Bacteriol 1995, 177:4I2I-4I30.

10. Sambrook J, Fritsch EF, Maniatis T: Molecular cloning: a laboratory manual 2nd edition. Cold Spring Harbor, NY: Cold Spring Harbor Laboratory Press; 1989.

II. Schmid MB, Roth JR: Genetic methods for analysis and manipulation of inversion mutations in bacteria. Genetics 1983, 105:517-537.

12. Datsenko KA, Wanner BL: One-step inactivation of chromosomal genes in Escherichia coli K-12 using PCR products. Proc Natl Acad Sci USA 2000, 97:6640-6645.

13. Porwollik S, Wong RM, McClelland M: Evolutionary genomics of Salmonella: gene acquisitions revealed by microarray analysis. Proc Natl Acad Sci USA 2002, 99:8956-8961.

14. Clarke MB, Sperandio V: Transcriptional regulation of flhDC by QseBC and sigma (FliA) in enterohaemorrhagic Escherichia coli. Mol Microbiol 2005, 57: 1734-1749.

15. Bansal T, Englert D, Lee J, Hegde M, Wood TK, Jayaraman A: Differential effects of epinephrine, norepinephrine, and indole on Escherichia coli O157:H7 chemotaxis, colonization, and gene expression. Infect Immun 2007, 75:4597-4607.

16. Chatterjee PK, Sternberg NL: A general genetic approach in Escherichia coli for determining the mechanism(s) of action of tumoricidal agents: application to DMP 840, a tumoricidal agent. Proc Natl Acad Sci USA 1995, 92:8950-8954.

17. Schaller A, Guo M, Gisanrin O, Zhang Y: Escherichia coli genes involved in resistance to pyrazinoic acid, the active component of the tuberculosis drug pyrazinamide. FEMS Microbiol Lett 2002, 2 I I:265-270.

18. Hong $Y$, Wang G, Maier RJ: The NADPH quinone reductase MdaB confers oxidative stress resistance to Helicobacter hepaticus. Microb Pathog 2008, 44:169-174.

19. Wang G, Alamuri P, Maier RJ: The diverse antioxidant systems of Helicobacter pylori. Mol Microbiol 2006, 6 I:847-860.

20. Wang G, Maier RJ: An NADPH quinone reductase of Helicobacter pylori plays an important role in oxidative stress resistance and host colonization. Infect Immun 2004, 72:1391-1396.

21. Clarke MB, Sperandio V: Transcriptional autoregulation by quorum sensing Escherichia coli regulators $B$ and $C$ (QseBC) in enterohaemorrhagic E. coli (EHEC). Mol Microbiol 2005, 58:44I-455.

22. Bearson BL, Bearson SM, Uthe JJ, Dowd SE, Houghton JO, Lee I, Toscano MJ, Lay DC Jr: Iron regulated genes of Salmonella enterica serovar Typhimurium in response to norepinephrine and the requirement of fepDGC for norepinephrineenhanced growth. Microbes Infect 2008, 10:807-816.
Publish with Bio Med Central and every scientist can read your work free of charge

"BioMed Central will be the most significant development for disseminating the results of biomedical research in our lifetime. "

Sir Paul Nurse, Cancer Research UK

Your research papers will be:

- available free of charge to the entire biomedical community

- peer reviewed and published immediately upon acceptance

- cited in PubMed and archived on PubMed Central

- yours - you keep the copyright
BioMedcentral 